\title{
ESTADO NUTRICIONAL DE TRABAJADORES BAJO TURNOS ROTATIVOS O PERMANENTES
}

\section{NUTRITIONAL STATE OF WORKERS UNDER STEADY OR ROTATING SHIFTS}

\author{
Marcela Ruiz de la F., María Trinidad Cifuentes M., Orieta Segura B., \\ Pamela Chavarria S., Ximena Sanhueza R. \\ Departamento de Nutrición y Salud Pública, Facultad de Ciencias de la Salud y de los Alimentos. \\ Universidad del Bío-Bío, Chillán, Chile.
}

\begin{abstract}
Restrictions of sleep predispose to experience, in the long term, excess malnutrition and non-transmissible chronic diseases. The objective of this research has to study the influence of working shifts on nutritional status lipid profile and fasting blood glucose (FBG). Study of a series of cases considered a total of 47 recruited workers from which 31 of them had a rotating shift (day and night) and 16 a steady shift (day). The nutritional assessment involved: BMI (Body Mass Index), waist and hip circumference, tricipital, bicipital, subescapular and suprailiac skinfolds. Sleep hours were studied with a weekly record, food intake was studied by means of 24-hours dietary recall. It was determined: total cholesterol, $c$-HDL, c-LDL, blood triglycerides and fasting blood glucose (FBG). The sample was composed by $87 \%$ women and $13 \%$ men, average age of 38,7 years $[19,0] v / s 44,7[25,2](p=0,420)$ in rotating and steady shifts respectively. The rotating shift did not show any significant differences compared to the steady shift in terms of anthropometric parameters except for subescapular skinfold that was significantly higher $(p=0,032)$ in relation to the macronutrients intake. Lipid profile and glycemia did not show important differences $(p=>0,05)$. The prevalence of the nutritional status was: rotating shift 35,4\% normal, 45,1\% overweight and 19,5\% obese. On the other hand, the steady shift showed: 43,7\% normal and 56,3\% overweight. Seniority only in the rotating shift showed a positive correlation of BMI $(r=0,436 p<0,005)$ body fat $(r=0,454 p<0,005)$ total cholesterol $(r=0,394$ $p<0,005) \mathrm{LDL}(r=0,484 p<0,005)$ and glycemia $(r=0,405 p<0,005)$. We concluded that workers of the rotating and steady shift did not show significant differences among variables studied except for the subescapular skinfold that was significantly higher in the rotating shift, that can be associated to predominantly central fat distribution. Key words: working shift, nutritional standing, lipid profile, sleep restriction.
\end{abstract}

Este trabajo fue recibido el 23 de Marzo de 2010 y aceptado para ser publicado el 12 de Noviembre de 2010.

\section{INTRODUCCIÓN}

El trabajo por sistema de turno nocturno es una práctica común en los hospitales debido a la necesidad de mantener una atención continua de los pacientes; sin embargo, este sistema de turno no está libre de riesgos para la salud de los trabajadores. Algunos estudios científicos señalan que el tipo de turno laboral influye en el estado de salud de las personas; es así como en los funcionarios con turnos nocturnos se ha descrito una mayor susceptibilidad a presentar de manera temprana perturbaciones en los hábitos alimentarios, reducción de la magnitud de las oscilaciones circadianas y ultradianas en las concentraciones de insulina y/o leptina (1), y un mayor riesgo nutricional de padecer estados de sobrepeso u obesidad (2); por otro lado y de manera más tardía, presentan un mayor riesgo de padecer enfermedades crónicas no transmisibles (resistencia insulina, diabetes, enfermedades cardíacas) (3) y estados de fatiga que contribuyen a niveles reducidos de actividad física.

Por consiguiente, y teniendo presente el gran número de trabajadores que deben realizar turnos de noche en el área de la salud, los objetivos planteados en este 
trabajo fueron: Evaluar el estado nutricional, cuantificar ingesta nutricional y analizar posibles factores de riesgo nutricional según dos tipos de turno: turno permanente, que incluye sólo turnos de día y turno rotativo que incluye turnos de día y noche, en el Servicio de Medicina del Hospital Clínico Herminda Martín de la ciudad de Chillán.

\section{SUJETOS Y MÉTODO}

La investigación se llevó a cabo mediante un estudio exploratorio del tipo de serie de casos. La población en estudio incluyó el $80 \%$ de los funcionarios, de ambos sexos, que realizaban turnos permanente o rotativos en el Servicio de Medicina del Hospital Clínico Herminda Martín de la ciudad de Chillán y que aceptaron participar de éste protocolo mediante la firma de un consentimiento informado. Se reclutó 47 casos, 31 funcionarios de turno rotativo, secuenciado en forma continua en un turno largo, de 08:00-20:00 hrs.; un turno mañana, de 08:00-13:00 hrs.; un turno de noche, de 20:00-08:00 hrs., seguido de dos días libres, y 16 funcionarios de turno permanente, cuya secuencia diaria era de lunes a viernes de 08:00 - 17:00 hrs. y los días sábados y domingo libres. El estudio fue aprobado por el Comité de Ética del hospital, y las evaluaciones aplicadas fueron las siguientes:

1. Mediciones antropométricas: Peso corporal a través de una balanza marca SECA modelo 713, con escala de 2 a $130 \mathrm{~kg}$ y precisión de $0,2 \mathrm{~kg}$; talla corporal a través de un tallímetro incorporado a la balanza con graduación mínima de $1 \mathrm{~mm}$; circunferencia del brazo por medio de una cinta métrica no distensible con graduación mínima de $1 \mathrm{~mm}$; pliegues cutáneos (bicipital, tricipital, subescapular y supraíliaco), utilizando un calibrador marca LANGE con sensibilidad de 1mm, graduación de 0-67 mm. (4-6).

2. A partir de la sumatoria de cuatro pliegues cutáneos (bicipital, tricipital, subescapular y supraíliaco) y la ecuación de Durnin y Womersley (7) se obtuvo la masa grasa $(\mathrm{MG})$ y por diferencia la masa libre de grasa (MLG).

3. La distribución de la grasa corporal se evaluó a través de la medición de la circunferencia de cintura y circunferencia de la cintura-cadera (8).

4. Se aplicó una encuesta nutricional tipo recordatorio de 24 horas de 3 días discontinuos (lunes, miércoles y viernes). Para determinar el aporte nutricional, se utilizó la tabla de composición química de los alimentos chilenos edición 1999.

5. Para evaluar el perfil lipídico y la glicemia se indicó un ayuno previo de 12 horas, extrayendo posteriormente $5 \mathrm{ml}$ de sangre venosa en ayunas.

6. Determinación de horas de sueño día: Mediante la consulta directa al funcionario del número de horas de sueño día, registrado a diario durante una semana.

Luego del trabajo de campo, se construyó una matriz de datos en el programa SPSS 17 para Windows, con la cual se describieron las variables continuas usando como medidas de tendencia central y dispersión, la media aritmética y desviación estándar previa comprobación de la normalidad, medida a través del test de ShapiroWilk. Cuando tal condición no se cumplió, se procedió a describir las variables mediante el uso de la mediana y rango interquartil. Se determinó la significancia estadística entre las variables con distribución normal a través de la prueba t- Student, y en aquellas sin distribución normal mediante la prueba de Mann-Whitney.

Con el objeto de analizar las variables de estudio según el tipo de turno, se realizó un análisis de regresión múltiple y se utilizó el coeficiente de correlación de Pearson (r), usando una prueba bilateral con nivel de significación del 5\%.

\section{RESULTADOS}

La distribución por género estuvo representada en $87 \%$ por mujeres y sólo en $13 \%$ por hombres. La distribución del sexo por turno laboral fue: turno rotativo, $90,4 \%$ mujeres y $9,6 \%$ hombres; turno permanente, $81,2 \%$ y $18,7 \%$ de mujeres y hombres, respectivamente. La actividad profesional de los funcionarios con turno permanente estuvo representada por 4 enfermeras universitarias, 8 auxiliares de servicio y 4 paramédicos. El turno rotativo estuvo conformado por 6 enfermeras universitarias y 25 técnicos paramédicos. La mediana de la antigüedad de los funcionarios con turno rotativo fue de 6,5 años [19,0] versus los funcionarios con turno permanente de 13 años [23]. Con relación al promedio semanal de horas de sueño día de los funcionarios, estas resultaron ser estadísticamente diferente, refiriendo el turno rotativo un promedio de $6,4 \pm 1,7 \mathrm{hrs}$. de sueño/ día, versus 7,4 $\pm 1,5 \mathrm{hrs}$. de sueño/día semanal señalada por el turno permanente (Mann-Whitney, $\mathrm{p}=0,035$ ).

La mediana de edad en años entre los grupos estudiados no resulto significativamente diferente $(\mathrm{p}=0,420)$, siendo en los funcionarios de turno rotativo de 38,7 años [19,0]; y en el turno laboral permanente de 44,7 años $[25,2]$.

La mediana del IMC (índice de masa corporal) obtenido de los funcionarios que trabajan con turno rotativo fue de $26,5 \mathrm{~kg} / \mathrm{m} 2[6,7] \mathrm{y}$, con turno permanente de $25,2 \mathrm{~kg} / \mathrm{m} 2[5,0]$, sin diferencia estadística significativa $(\mathrm{p}=0,393)$; presentando sólo el turno rotativo una correlación positiva entre la antigüedad del turno y el IMC $(\mathrm{r}=0,436 \mathrm{p}<0,05)$. La clasificación del estado nutricional según IMC de los funcionarios con turno rotativo fue 
el 29,0\% de las funcionarias de sexo femenino con turno rotativo y el $18,7 \%$ con turno permanente presentaron valores mayores a $88 \mathrm{~cm}$ de cintura. Por el contrario, en los funcionarios vaones, ninguno de los grupos estudiados mostró una circunferencia de cintura mayor a $102 \mathrm{~cm}$, asociados a alto riesgo cardiovascular (tabla 1).

El estudio general de la ingesta dietaría de los funcionarios con turno laboral rotativo y permanente, reveló que el consumo promedio día no mostró diferencias estadísticamente significativas en cada uno de ellos (tabla 2). Respecto a la ingesta calórica en ambos grupos estudiados, el sexo masculino presentó numéricamente mayores valores, resultando en el turno rotativo la ingesta de las mujeres de 2106,3+554,68 cal/día versus los hombres de 2332,40+88,86; y en el turno permanente de $1903,77+799,84$ versus $2543,20+1024$ cal/día, respectivamente. La ingesta entre los grupos analizados de ácidos grasos omega $3(\mathrm{p}=0,636)$, omega $6(\mathrm{p}=0,678)$ y colesterol dietario $(\mathrm{p}=0,729)$, tampoco mostró diferencias estadísticamente significativas (tabla 2).

La tabla 3, muestra en general que ambos grupos estudiados presentaron niveles promedios de colesterol

\section{TABLA 2}

\section{Ingesta nutricional según turno laboral de funcionarios del Servicio de Medicina Hospital Herminda Martín, Chillán.}

\begin{tabular}{|c|c|c|c|c|}
\hline \multirow[t]{2}{*}{ VARIABLES } & \multirow{2}{*}{$\begin{array}{c}\text { Shapiro-Wilk Test } \\
\text { P-Value }\end{array}$} & \multicolumn{2}{|c|}{ TURNO } & \multirow[b]{2}{*}{$\boldsymbol{P}$} \\
\hline & & Rotativo (n=31) & Permanente $(n=16)$ & \\
\hline Kcal/día & $0,323+\dagger$ & $2121,38+538,54$ & $2023,67+847,50$ & 0,635 \\
\hline Proteínas (g) & $0,451 \dagger \dagger$ & $77,96+18,20$ & $72,74+32,06$ & 0,483 \\
\hline Hidratos de carbono (g) & $0,051 \dagger \dagger$ & $298,41+79,84$ & $276,22+125,98$ & 0,469 \\
\hline Lípidos (g) & $0,713+\dagger$ & $68,34+21,46$ & $64,85+31,43$ & 0,659 \\
\hline Ácidos grasos saturados (g) & $0,093+\dagger$ & $19,53+6,11$ & $18,34+10,82$ & 0,636 \\
\hline Ácidos grasos monoinsaturados (g) & $0,025 \dagger$ & $19,70[14,4]$ & $18,67[12,03]$ & 0,596 \\
\hline Ácidos grasos poliinsaturados (g) & $0,126+\dagger$ & $17,36+7,43$ & $17,89+9,76$ & 0,837 \\
\hline n6 (g) & $0,001 \dagger$ & $11,26[2,30]$ & $9,73[2,97]$ & 0,678 \\
\hline $\mathrm{n} 3(\mathrm{~g})$ & $<0,001 \dagger$ & $0,38[0,28]$ & $0,43[0,93]$ & 0,636 \\
\hline Colesterol total (mg) & $0,005 \dagger$ & $182,92[60,0]$ & $184,88[49,5]$ & 0,729 \\
\hline
\end{tabular}

\section{TABLA 3}

Perfil lipídico y glicemia de los trabajadores de la salud con diferente turno laboral

\begin{tabular}{|c|c|c|c|c|}
\hline VARIABLES & $\begin{array}{c}\text { Shapiro-Wilk Test } \\
\text { P-Value }\end{array}$ & Rotativo (n=31) & $\begin{array}{c}\text { TURNO } \\
\text { Permanente }(n=16)\end{array}$ & $P$ \\
\hline Col-total en sangre & $0,995+\dagger$ & $213,43+43,02$ & $203,63+42,48$ & 0,46 \\
\hline Triglicéridos en sangre & $<0,001 \dagger$ & $106,50[106,0]$ & $102,00[118,50]$ & 0,22 \\
\hline Col-HDL en sangre & $0,151 \dagger \dagger$ & $42,87+8,37$ & $43,13+11,86$ & 0,93 \\
\hline Col-LDL en sangre & $0,046 \dagger$ & $144,00[52,0]$ & $123,50[35,5]$ & 0,49 \\
\hline Glicemia en sangre & $<0,001 \dagger$ & $87,50[7]$ & $88,50[15]$ & 0,84 \\
\hline
\end{tabular}

$\dagger$ Variable no se distribuye normal, se describe a través de la Mediana y el Rango: $M_{4} \pm[$ rango $]$ (prueba de Mann-Whitney)

$\dagger$ Variable se distribuye normal, se describe mediante Media aritmética y Desviación estándar: $x \pm s$ (prueba t-Student). 
total superior al nivel de normalidad $(<200 \mathrm{mg} / \mathrm{dl})$, sin diferencias estadísticamente significativas $(\mathrm{p}=0,463)$. El $64,5 \%$ de los funcionarios de turno rotativo clasificó con niveles de colesterol sérico mayores a lo normal, a diferencia del $50 \%$ observado en los funcionarios de turno permanente, pero sin diferencia significativa $(\mathrm{p}=0,14)$.

Respecto a los valores de triglicéridos, no hubo diferencia significativa entre los grupos estudiados $(\mathrm{p}=0,226)$. Los valores promedio de colesterol HDL en el sexo femenino, fueron superiores a los recomendados $(>50 \mathrm{mg} / \mathrm{dl})$ en un $78,5 \%$ en el turno rotativo versus $69,2 \%$ en el turno permanente, respecto al sexo masculino ambos grupos presentaron igualmente valores superiores a lo recomendado ( $>40 \mathrm{mg} / \mathrm{dl})$ en $66,6 \%$. El colesterol LDL no presentó diferencia significativa entre los grupos estudiados $(\mathrm{p}=0,499)$. El 54,8\% de los funcionarios con turno rotativo, versus el $37,5 \%$ del turno permanente, clasificaron con valores de LDL superiores a lo recomendado ( $\geq 140 \mathrm{mg} / \mathrm{dl}$ ), aunque sin diferencia significativa $(\mathrm{p}=0,41)$.

Los niveles de glicemia basal no mostraron diferencias significativas entre los grupos estudiados, presentando valores de $87,50 \mathrm{mg} / \mathrm{dl}[7,0]$ los de turno rotativo, versus $88,5 \mathrm{mg} / \mathrm{dl}[15,0]$ los con turno permanente $(\mathrm{p}=0,844)$. El número de funcionarios por grupo estudiado que presentaron glicemias anormales $(>100$ $\mathrm{mg} / \mathrm{dl}$ ) fue de un 22,5\% de los funcionarios (7) con turno rotativo, y de $12,5 \%$ de funcionarios (2) con turno permanente, igualmente sin diferencia significativa $(\mathrm{p}=0,40)$.

Se estudió mediante análisis de regresión múltiple la asociación entre la variable horas de sueño, con las variables perfil lipídico, glicemia, porcentaje de grasa corporal y circunferencia de cintura, no encontrándose asociación entre ellas, sin embargo de manera exploratoria se observaron algunos coeficientes de correlación significativos, descritos en la tabla 4. Al correlacionar las variables de horas de sueño al día y parámetros antropométricos en los grupos estudiados, sólo el turno permanente mostró una correlación positiva entre horas de sueño día con la relación cintura/cadera $(0,755$ $\mathrm{p}<0,01)$. La variable antigüedad laboral del turno rotativo se correlacionó positivamente con el IMC $(0,436$ $\mathrm{p}<0,05)$, con el porcentaje de masa grasa $(0,454 \mathrm{p}<0,05)$ y negativamente con el porcentaje de masa libre de grasa

\section{TABLA 4}

\section{Correlaciones entre el promedio semanal de horas de sueño y la antigüedad en el turno según variables antropométricas y exámenes de laboratorios a funcionarios con turno rotativo y permanente.}

\begin{tabular}{|c|c|c|c|}
\hline & Variables & $\begin{array}{c}\text { Horas de sueño } \\
\text { Rotativo / permanente }\end{array}$ & $\begin{array}{c}\text { Antigüedad turno } \\
\text { Rotativo / Permanente }\end{array}$ \\
\hline & $\mathrm{IMC}^{*}$ & $-0,06 / 0,55$ & $0,43+\div / 0,16$ \\
\hline & $\mathrm{CC} \ddagger$ & $-0,03 / 0,75^{* * *} *$ & $0,27 /-0,01$ \\
\hline & $\mathrm{MGC}_{1}^{\prime}$ & $-0,30 / 0,16$ & $0,45+\% / 0,30$ \\
\hline & $\mathrm{MLG}^{* *}$ & $0,30 /-0,16$ & $-0,45 \div \div /-0,30$ \\
\hline & $\mathrm{Tg} \dagger \dagger$ & $0,38 \div \ddagger / 0,15$ & $-0,13 / 0,22$ \\
\hline & Col-Total & $0,05 /-0,25$ & $0,39+\div / 0,38$ \\
\hline & Col-HDL & $-0,31 /-0,29$ & $0,37 \div \div / 0,05$ \\
\hline & Col-LDL & $-0,33 /-0,20$ & $0,48 * * * / 0,30$ \\
\hline & Glicemia & $-0,18 / 0,26$ & $0,40+\$ / 0,09$ \\
\hline $\begin{array}{l}\text { El an } \\
\text { y } 16 \\
\text { Corr } \\
+\dagger \\
* * * \\
* \\
\dagger \\
\square \\
* * \\
\dagger \dagger\end{array}$ & $\begin{array}{l}\text { Inálisis de las variables ant } \\
5 \text { de turno permanente. } \\
\text { relación Pearson } \\
\mathrm{p}<0,05 ; \\
\mathrm{p}<0,01 \\
\text { Índice de Masa Corporal } \\
\text { Circunferencia cadera } \\
\text { Masa grasa corporal } \\
\text { Masa libre de grasa } \\
\text { Triglicéridos }\end{array}$ & de laboratorio estuvo confor & uncionarios de turno rotativo \\
\hline
\end{tabular}


corporal $(-0,454 \mathrm{p}<0,05)$. Al determinar correlaciones entre las horas de sueño día según turno laboral y perfil lipídico, sólo el turno rotativo correlacionó positivamente el promedio de horas de sueño día ( 6,4 horas/día) con los niveles de triglicéridos $(0,382 \mathrm{p}<0,05)$. Asimismo sólo el turno rotativo correlacionó positivamente la antigüedad laboral con los niveles de colesterol total $(0,394$ $\mathrm{p}<0,05)$, colesterol HDL $(0,378 \mathrm{p}<0,05)$ y colesterol LDL $(0,484 \mathrm{p}<0,01)$. El turno rotativo también mostró una correlación positiva entre antigüedad laboral y los niveles de glicemia en ayuno $(0,405 ; \mathrm{P}<0,05)$.

\section{DISCUSIÓN}

Existe evidencia científica que fundamenta la asociación entre el largo dormir ( $>8 \mathrm{hrs} /$ día) y el incremento de morbilidad y mortalidad en el ser humano $(9,10)$; no obstante, también se ha descrito una asociación entre el corto dormir ( $<7$ hrs./día) con algunos efectos adversos para la salud, explicados por la alteración de los ritmos circadianos o biológicos, los cuales son responsables de modular diversas variables fisiológicas de nuestro organismo (hormonales, frecuencia cardíaca, temperatura corporal, entre otros.). La alteración de estos ritmos biológicos frente a restricciones del sueño, facilitan la mayor prevalencia de eventos fisiológicos negativos en el organismo, entre ellos el riesgo de padecer malnutrición por exceso, enfermedades crónicas no transmisibles $(3,11,12)$ y cáncer a largo plazo (13). Algunos estudios de corte transversal, han mostrado un consistente riesgo de padecer obesidad entre los adultos de corto dormir; sin embargo, debido a algunas limitaciones metodológicas de las investigaciones realizadas, no se ha podido establecer una inferencia causal entre la restricción del sueño y la obesidad $(14,15)$.

La asociación negativa entre la restricción del sueño y el desarrollo de sobrepeso y obesidad en el ser humano, se ha relacionado también con el número de horas de sueño al día reducidas; es así como se ha referido que una rebaja del sueño de alrededor de 6 horas /día, facilitaría los efectos adversos antes descritos (16-18), característica observada parcialmente en los funcionarios de turno rotativo estudiados, quienes además llevaban en promedio más de 6,5 años de antigüedad laboral. La ausencia de estudios nacionales con esta temática motivó el desarrollo de esta investigación.

El análisis de la mayoría de los parámetros antropométricos no fue estadísticamente diferente entre los grupos estudiados $(p>0,05)$, sin embargo la prevalencia de malnutrición por exceso, de sobrepeso y obesidad, fue mayor en el turno rotativo, el que además mostró una correlación positiva entre los años de antigüedad laboral y el IMC ( $\mathrm{p}<0,05)$, aún siendo la mediana de los años de antigüedad laboral de los funcionarios estudiados considerablemente menor a los del turno rotativo. En relación a lo anterior diversas investigaciones han asociado la restricción de horas de sueño a un aumento del IMC (19-23).

La antigüedad del turno rotativo, se correlacionó positivamente con el porcentaje de grasa corporal $(\mathrm{r}=0,454$ $\mathrm{p}<0,05)$, y negativamente con la masa libre de grasa $(\mathrm{r}=-0,454 \mathrm{p}<0,05)$, sugiriendo algún efecto negativo de la restricción del sueño por día, en la composición corporal de los individuos reclutados.

La obesidad es considerada un factor de riesgo independiente de padecer cardiopatías coronarias; sin embargo, más que el contenido de grasa corporal total es la distribución del tipo androide la que predice dicho riesgo. De acuerdo a lo anterior, fue importante conocer la distribución del tejido adiposo de los funcionarios, teniendo en cuenta los diversos estudios epidemiológicos que han determinado que la distribución de la grasa corporal es más importante que la cantidad de grasa total, desde un punto de vista mórbido. Respecto a lo anterior los resultados de las mediciones de circunferencia de cintura y relación cintura-cadera, no mostraron diferencias significativas entre los grupos estudiados; sin embargo fueron las mujeres del turno rotativo quienes presentaron una mayor prevalencia de circunferencia de cintura de alto riesgo cardiovascular $(>88 \mathrm{~cm})$. Por otro lado, la medición del pliegue cutáneo subescapular, que es asociado per se a obesidad troncal $(24,25)$ y a enfermedad cardiovascular (22), resultó significativamente superior $(\mathrm{p}<0,05)$ en los funcionarios con turno rotativo, señalando por lo tanto una distribución de grasa corporal predominantemente central, la que se relacionó a un mayor riesgo de enfermedades cardiovasculares, dislipidemias y ciertos tipos de cáncer (26). Las observaciones encontrados a nivel local, fueron coincidentes con otros estudios internacionales que han relacionado la restricción del sueño con la distribución de grasa corporal predominantemente central $(27,28)$.

La desregulación del apetito relacionada con la restricción del sueño día (29), se fundamenta en algunos cambios hormonales, específicamente en lo que se refiere a la disminución de los niveles de leptina, hormona que actúa inhibiendo el apetito a nivel hipotalámico, y por el aumento en los niveles de ghrelina, hormona cuya función es estimular ciertas neuronas hipotalámicas que ocasionan aumento del apetito $(1,30)$. En esta investigación, el estudio de la dieta a través de tres días discontinuos de la semana, no arrojó diferencias significativas en la ingesta nutricional entre los individuos con turno rotativo y permanente. 
En el estudio, la mediana de los niveles de glicemia en ayuno no fue estadísticamente diferente entre los grupos estudiados $(\mathrm{p}=0,844)$, sin embargo se observó la existencia de una correlación positiva entre los años de antigüedad laboral sometidos a restricciones del sueño del turno rotativo y los niveles de glicemia en ayuno $(\mathrm{r}=0,405 \mathrm{p}=<0,05)$, sugiriendo de este modo algún grado de alteración en el metabolismo de la glucosa; semejante a lo encontrado en otras investigaciones internacionales que han descrito que la restricción del sueño día se asocia al deterioro del metabolismo de la glucosa e incremento del riesgo de diabetes mellitus, independientemente de los cambios del IMC $(29,31)$.

Estudios internacionales señalan que los funcionarios sometidos a turnos laborales que incluyen la realización de turnos nocturnos, presentan una mayor prevalencia de dislipidemia $(32,33)$, situación que no se observó en los individuos estudiados. No obstante, en los funcionarios sometidos a turno nocturnos, fue donde la variable antigüedad laboral, en analogía a los años sometidos a alteraciones del ciclo de sueño vigilia, se correlacionó positivamente con los valores de colesterol total $(\mathrm{r}=0,394 \mathrm{p}<0,05)$ y colesterol $\mathrm{LDL}$ $(\mathrm{r}=0,484 \mathrm{p}<0,05)$.

En relación a lo anterior, los sistemas de turnos laborales se asocian a una variedad de problemas fisiológicos que pueden afectar la salud de los trabajadores (34); situación observada de algún modo en este estudio de tipo exploratorio, que mostró en los funcionarios estudiados sometidos a restricciones del sueño, cierta tendencia a una mayor distribución de grasa corporal de localización central y algún grado de correlación significativa $(\mathrm{p}<0,05)$ entre la antigüedad laboral del turno rotativo con el porcentaje de grasa corporal, el perfil lipídico y los niveles de glicemia en ayuno.

La restricción del sueño es un factor de riesgo modificable (35); sin embargo existen situaciones laborales donde la realización de turnos rotativos, que incluyen turnos de noche, se hace imprescindible, como es el caso de los hospitales donde se requiere mantener un proceso continuo de 24 horas al día; es en esa situación donde creemos importante incorporar en la elaboración de turnos rotativos, medidas que faciliten la recuperación saludable del sueño en los trabajadores $(36,37)$.

Por último nos parece necesario, en primer lugar la realización de más investigaciones locales de igual temática que verifiquen las observaciones encontradas, y en segundo lugar, considerar en los trabajadores sometidos a turnos nocturnos, la práctica de controles periódicos en salud que permitan determinar posibles alteraciones, mediante el estudio de hábitos alimentarios, estado ponderal; de manera de prevenir y/o actuar oportunamente en el tratamiento de las enfermedades asociadas a los estilos de vida adoptados.

\section{RESUMEN}

Restricciones del sueño predisponen a padecer, a largo plazo, malnutrición por exceso y enfermedades crónicas no transmisibles. El objetivo fue estudiar la influencia del turno laboral sobre estado nutricional, perfil lipídico y glicemia en ayuno. Estudio exploratorio del tipo de serie de casos, 47 reclutados; 31 turnos rotativos (día y noche), y 16 turno permanente (día). La evaluación nutricional se efectuó mediante el IMC, la circunferencia cintura-cadera y los pliegues trícipital, bícipital, subescapular y suprailiaco. Se estudió las horas sueño mediante un registro semanal, la ingesta dietética por encuesta de recordatorio de 24 horas. Se determinó el colesterol total, c-HDL, c-LDL, triglicéridos séricos y glicemia. La muestra estuvo representada en un $87 \%$ mujeres y $13 \%$ por hombres, cuya mediana de edad fue de 38,7 años [19,0] y 44,7[25,2] $(\mathrm{p}=0,420)$ en turno rotativo y permanente, respectivamente. El turno rotativo no mostró diferencias significativas respecto al turno permanente en los parámetros antropométricos a excepción, del pliegue subescapular que fue significativamente superior $(\mathrm{p}=0,032)$, respecto a ingesta de macronutrientes, perfil lipídico y glicemia tampoco mostró diferencias significativas $(\mathrm{p}=>0,05)$. La prevalencia del estado nutricional en el turno rotativo fue en $35,4 \%$ normal, en $45,1 \%$ sobrepeso y en $19,5 \%$ obesidad, y turno permanente: $43,7 \%$ normal y $56,3 \%$ sobrepeso. La antigüedad laboral sólo en el turno rotativo se correlacionó positivamente con IMC $(\mathrm{r}=0,436 \mathrm{p}<0,005)$, grasa corporal $(\mathrm{r}=0,454 \mathrm{p}<0,005)$, el colesterol total $(r=0,394 \mathrm{p}<0,005)$ el LDL $(r=0,484$ $\mathrm{p}<0,005)$ y la glicemia $(\mathrm{r}=0,405 \mathrm{p}<0,005)$. En conclusión, los funcionarios del turno rotativo y permanente no mostraron diferencias significativas entre las variables estudiadas, a excepción del pliegue subescapular que fue significativamente mayor en el turno rotativo, lo que podría asociarse a la distribución de la grasa predominantemente central.

Palabras clave: turno laboral, estado nutricional, perfil lipídico, restricción de sueño.

Dirigir la correspondencia a:

Prof. Marcela Ruiz De La Fuente

Nutricionista

Magíster en Nutrición Clínica Adulto

Departamento de Nutrición y Salud Pública.

Universidad del Bío-Bío, sede Chillán

Teléfono: (56-42) 253235

Email:marcelaruiz@ubiobio.cl 
Agradecimientos: Los autores agradecemos al Director Médico del Hospital Clínico Herminda Martín de Chillán Dr. Rodrigo Avendaño Brandeis, y a quienes fueron en ese entonces, jefe del Servicio de Medicina Dra. Margarita Valenzuela y enfermera jefe del Servicio de Medicina Sra. Rosa Muñoz Cabezas, por haber facilitado la realización de esta investigación. También agradecemos las sugerencias técnicas del nutricionista doctor Erick Díaz Bustos y de la doctora María Pía de la Maza Cave.

\section{BIBLIOGRAFÍA}

1. Copinschi G. Metabolic and endocrine effects of sleep deprivation. Essent Psychopharmacol. 2005;6(6):341-7.

2. Stamatakis KA, Brownson RC. Sleep duration and obesity-related risk factores in the rural Midwest. Prev Med. 2008;46(5):439-44.

3. Wolk R, Gami AS, Garcia- Touchard A el al. Sleep and cardiovascular disease. Curr Probl Cardiol. 2005; 30(12):625-62.

4. Gordon C, Cameron W, Rochet A. Stature, recumbent lenght, and weight. In: Lohman T, Roche A, Martorell R. Anthropometric standardization reference manual. Abidged Edition. 1988:3-8.

5. Callaway W, Cameron, Bouchard C, Himes J, Lohman T, Martín A, et al. Circumferences. In: Lohman T, Roche A, Martorell R. Anthropometric standardization reference manual. Abidged Edition. 1988:p 39-54.

6. Harrison G, Buskirk E, Carter J, Johnston F, Lohman T, Pollock M, et al. Skinfold thicknesses and measurement technique. In: Lohman T, Roche A, Martorell R. Anthropometric standardization reference manual.. Abidged Edition. 1988:pp 55-70.

7. Durnin, JV, Womersley, J. Body fat assessed from total body density and its estimation from skinfold thickness: measurements on 481 men and women aged from 16 to 72 years Br J Nutr. 1974; 32:77-97.

8. Obesity: preventing and managing the global epedimic. Report of a WHO consultation on obesity. Geneva, 5 - 5 june 1997.

9. Kripke DF, Garfinkel L, Wingard DL at el. Mortality associated with sleep duration and insomnia. Arch Gen Pssychiartry. 2002;59:131-6.

10. Ayas NT, White DP, Al-Delaimy WK, et al. A prospective study of selfreported sleep duration and incident diabetes in women. Diabetes Care. 2003;26:380-4

11. Miller MA, Cappucio FP. Inflamation, sleep, obesity and cardiovascular disease. Curr Vasc Pharmacol. 2007; 5(2):93-102.
12. Yaggi HK, Araijo AB, McKinlay JB. Sleep duration as risk factor for the development of type 2 diabetes. Diabetes fare 2006:29:657-61.

13. Davis S, Mirick DK. Circadian disruption, shift work and the risk of cancer a summary of the evidence and studies in Seattle. Cancer Causes Control. 2006;17(4):539-45.

14. Francesco P, Cappuccio, MD., Frances M. T et al. Meta-analisis of short sleep duration and obesity in children and adults. Sleep.2008;1;31(5):619-626.

15. Gottlieb DJ, Redline S, Nieto FJ, et al. Association of usual sleep duration with hypertension: the Sleep Heart Health Study. Sleep 2006;29:1009-14.

16. López-García E, Faubel R, León-Munoz L, et al. Sleep durationt, general and abdominal obesity, and weight change among the older adult population of Spain Am LClin Nutr 2008; 87(2);310-6

17. Shigeta $\mathrm{H}$ Shigeta $\mathrm{M}$, Nakazawa A Nakamura N, Yoshikawa T. Lifestyle, obesity, and insulin resistance. Diabetes Care.2001;24 (3):608.

18. Courtot M, Ruidavest JB, Marquié JC, et al. Environmental factors associated with body mass index in a poplation of Southern France. Eur J Cardiovasc Prev Rehabil. 2004;11(4):291-7.

19. Park YJ, Lee Wc, Yim HW, Park YM. The association between sleep and obesity in Korean adults. J Prev Med Pub Health. 2007;40(6):454-60.

20. Rontoyanni VG, Baic S, Cooper AR. Association between nocturnal sleep duration, body fatness, and dietary intake in Greek women. Nutrition. 2007;23 (11-12):773-7.

21. Sing M, Drake CL, Roehrs T, Hudgel DW, Roth T. The association between obesity and short sleep duration: a population- based study. J Clin Sleep Med.2005 15;1(4):357-63.

22. Patel Sr, malhotra A, White DP, Gottlieb DJ, $\mathrm{Hu}$ FB. Association between reduced sleep and weight gain in women. Am J Epidemiol. 2006; 15; 164(10):947-54.

23. Gangwisch JE, Malaspina D, Boden-Albala B, Heymsfield SB. Inadequate sleep as a risk factor for obesity: analyses of the NHANES I. Sleep.2005;1;28(10):1289-1296.

24. Wells JC, Victoria CG. Indices of whole-body and central adiposity for evaluating the metabolic load of obesity. Int J Obes (Lond). 2005;29(5):483-9.

25. David Tanne, MD; Jack H. Medalie, MD Uri Goldbourt, PhD. Body Fat Distribution and Long-Term Risk of Stroke Mortality. Stroke. 2005;36:1021-1025

26. Bouchard, C., Perusse, L., LE, Blanc., Trembley, A., Theriault G. Inheritance of the amount and distribution of human body fat. Int J Obesity 
27.

1998:12:205-15

Park SE, Kim HM, Kim DH, Kim J., Cha BS, Kim
DJ. The association between sleep duration and general and abdominal obesity in Koreans: data from the Korean National Health and Nurrition Examination Survey, 2001 and 2005. Obesity. 2009; 17(4):767-71.

28. Yu Y, Lu BS, Wang B et al. Short sleep duration and adiposity in Chinese adolescentes. Sleep. 2007 1;30(12):1688-97.

29. Knutson K. , Spiegel K., Penev P., et al. The metabolic consequences of sleep deprivation. Sleep Med Rev.2007;11(3): 163-178.

30. Shahrad Taheri, Ling Lin, Diane Austin et al. Short sleep duration is associated with reduced leptin, elevated ghrelin, and increased body mass index. PLoS Med.2004;1(3):e62.

31. Spiegel K. et al. Sleep loss: a novel risk factor for insulin resistance and Type 2 diabetes. J Applied Physiol. 2005; 99:2008-19.

32. Karlsson B, Knutsson A \& Lindahl B. Is there an association between shift work and having a metabolic syndrome? Results from a population based study of 27485 people. Occup Environ Med .2001;58: 747-52.

33. De Bacquer D, Van Risseghem M, Clays E, Kittel F, De Backer G, and Braeckman L. Rotating shift work and the metabolic syndrome: a prospective study. Int. J. Epidemiol. 2009; 38(3):854-5.

34. Akeerstedt T. Physiological and phychophysiological effects of shift work. Scand J Work Environ Health. 1990.16(Supl. 1):67-73.

35. Hasler G, Buysse D, Klaghohr R, Gamma A, Ajdacic Vladeta A. The association between short sleep duration and obesity in young adults: a 13- year prospective study Sleep. 2004; 27(4):661-6.

36. Berger AM Hobbs BB. Impact of shift work on the health and safety of nurses and patients. Clin J Oncol Nurs. 2006;10(4):465-71

37. Knauth P, Hornberger S. Preventive and compensatory measure for shift workers. Occupational Med. 2003;53:109-16. 